ROTA'S BASIS CONJECTURE FOR PAVING MATROIDS

\author{
Jim Geelen and Peter J Humphries \\ Department of Mathematics and Statistics \\ University of Canterbury \\ Private Bag 4800 \\ Christchurch, New Zealand
}

Report Number: UCDMS2006/6

JUNE 2006 


\title{
ROTA'S BASIS CONJECTURE FOR PAVING MATROIDS
}

\author{
JIM GEELEN AND PETER J. HUMPHRIES
}

\begin{abstract}
Rota conjectured that, given $n$ disjoint bases of a rank- $n$ matroid $M$, there are $n$ disjoint transversals of these bases that are all bases of $M$. We prove a stronger statement for the class of paving matroids.
\end{abstract}

\section{Introduction}

We prove the following theorem.

Theorem 1.1. Let $B_{1}, \ldots, B_{n}$ be disjoint sets of size $n \geq 3$ and let $M_{1}, \ldots, M_{n}$ be rank-n paving matroids on $\bigcup_{i} B_{i}$ such that $B_{i}$ is a basis of $M_{i}$ for each $i \in\{1, \ldots, n\}$. Then there exist $n$ disjoint transversals $A_{1}, \ldots, A_{n}$ of $B_{1}, \ldots, B_{n}$ such that $A_{i}$ is a basis of $M_{i}$ for each $i \in$ $\{1, \ldots, n\}$.

A paving matroid $M$ is a matroid in which each circuit has size $r(M)$ or $r(M)+1$, where $r(M)$ is the rank of $M$. Theorem 1.1 implies Rota's basis conjecture for paving matroids.

Conjecture 1.2 (Rota). Given $n$ disjoint bases $B_{1}, \ldots, B_{n}$ in a rank-n matroid $M$, there exist $n$ disjoint transversals $A_{1}, \ldots, A_{n}$ of $B_{1}, \ldots, B_{n}$ that are all bases of $M$.

For $n=2$, Conjecture 1.2 follows immediately from basis exchange in matroids. Chan [2] proved the conjecture for $n=3$. Wild [9] proved a stronger conjecture for the class of strongly base-orderable matroids, while more recently a slightly weaker result was proved for a general matroid (Ponomarenko [8]). Further partial results may be found in [1], [3], [4], [5] and [9].

Theorem 1.1 fails for both $n=2$ and matroids in general. When $n=2$, if we take $\mathcal{B}\left(M_{1}\right)=\{\{e, f\},\{e, g\},\{f, h\},\{g, h\}\}$ and $\mathcal{B}\left(M_{2}\right)=$

Date: June 2, 2006.

1991 Mathematics Subject Classification. 05B35.

Key words and phrases. Rota's basis conjecture, paving matroids.

This research was partially supported by grants from the Natural Sciences and Engineering Research Council of Canada and the New Zealand Marsden Fund. 
$\{\{e, f\},\{e, h\},\{f, g\},\{g, h\}\}$, then $\{e, f\},\{g, h\}$ is the only pair of disjoint bases. In the second instance, if one of the matroids $M_{i}$ has all the $x \in E-B_{i}$ as loops, then there are no independent transversals of $B_{1}, \ldots, B_{n}$ in $M_{i}$.

The remainder of this paper is taken up with the proof of the theorem. In Section 2, we prove that Theorem 1.1 holds when $n=3$. This result is then used in Section 3 to prove the theorem inductively.

\section{THE CASE $n=3$}

For basic concepts in matroid theory, the reader is referred to Oxley [7]. We follow the same notation as Oxley throughout this paper.

A closed set in a matroid is commonly known as a flat. We will primarily be interested in rank-2 flats, or lines. In the proof of Theorem 2.1, we make frequent use of the fact that if $r_{M}(X)=r_{M}(Y)=2$ and $|X \cap Y| \geq 2$, then $X$ and $Y$ are contained in the same line in $M$.

Theorem 2.1. Theorem 1.1 holds for $n=3$.

Proof. If we assume the theorem is false, then there exist bases $B_{1}=\left\{a_{1}, a_{2}, a_{3}\right\}, B_{2}=\left\{b_{1}, b_{2}, b_{3}\right\}, B_{3}=\left\{c_{1}, c_{2}, c_{3}\right\}$ of rank-3 paving matroids $M_{1}, M_{2}, M_{3}$ respectively, with common ground set $E=$ $B_{1} \cup B_{2} \cup B_{3}$, that provide a counterexample. The rank of a set $X$ in a matroid $M_{i}$ will be denoted by $r_{i}(X)$ and the closure by $\operatorname{cl}_{i}(X)$. A three-element subset of $E$ will be called a transversal if it meets each of $B_{1}, B_{2}$, and $B_{3}$. Note that we may assume that every non-trivial line in each matroid contains a transversal, since all non-trivial lines not containing a transversal may be relaxed to provide an alternative counterexample (see [7], Section 1.5, Exercise 3).

2.1.1. Let $X \subseteq E$ be a set that meets each of $B_{1}, B_{2}, B_{3}$. If $r_{i}(X)=3$, then $X$ contains an $M_{i}$-independent transversal.

Subproof. Let $T \subseteq X$ be a transversal of $B_{1}, B_{2}, B_{3}$, and suppose that $T$ is $M_{i}$-dependent. Then since $r_{i}(X)=3$, there is some $e \in X$ such that $e \notin \mathrm{cl}_{i}(T)$. Without loss of generality, $e \in B_{1}$, so let $f$ be the unique element in $T \cap B_{1}$. Then $r_{i}((T-f) \cup e)=3$, and we are done.

2.1.2. If no $M_{1}$-dependent transversal contains both $a_{1}$ and $b_{1}$, then there exists $e \in B_{3}$ such that $r_{2}\left(E-\left\{a_{1}, b_{1}, e\right\}\right)=2$.

Subproof. For each $a \in B_{1}$ and $b \in B_{2}$, there exists $c \in B_{3}$ such that $\{a, b, c\}$ is $M_{3}$-independent (since $r_{3}\left(B_{3}\right)=3$ ). In particular, there exist $e, f, g \in B_{3}$ such that $\left\{a_{2}, b_{3}, e\right\},\left\{a_{3}, b_{3}, f\right\}$, and $\left\{a_{2}, b_{2}, g\right\}$ are $M_{3^{-}}$ independent. Then, by 2.1.1, $\left\{a_{3}, b_{2}\right\} \cup\left(B_{3}-\{e\}\right),\left\{a_{2}, b_{2}\right\} \cup\left(B_{3}-\{f\}\right)$, and $\left\{a_{3}, b_{3}\right\} \cup\left(B_{3}-\{g\}\right)$ all have rank 2 in $M_{2}$ (since otherwise we would 
find the required partition into transversals). The second and third of these sets both have two points in common with the first, and so they are all contained in a common line in $M_{2}$.

Suppose $M_{1}$ has a line $L$ containing at least seven elements. Since $r_{1}\left(B_{1}\right)=3,\left|L-B_{1}\right| \geq 5$. Up to symmetry, we may assume that $b_{1}, b_{2}, c_{1}, c_{2}, c_{3} \in L$ and that $a_{1} \notin \mathrm{cl}_{1}(L)$. Now neither $\left\{a_{1}, b_{1}\right\}$ nor $\left\{a_{1}, b_{2}\right\}$ is in an $M_{1}$-dependent transversal. So by 2.1.2 $r_{2}\left(\left\{a_{2}, a_{3}, b_{2}, b_{3}\right\}\right)=r_{2}\left(\left\{a_{2}, a_{3}, b_{1}, b_{3}\right\}\right)=2$, contradicting the fact that $r_{2}\left(B_{2}\right)=3$. Thus none of $M_{1}, M_{2}$, and $M_{3}$ contain a line on seven or more elements.

2.1.3. Every pair $e \in B_{i}, f \notin B_{i}$ is contained in some $M_{i}$-dependent transversal.

Subproof. Suppose no $M_{1}$-dependent transversal contains both $a_{1}$ and $b_{1}$. Then, by 2.1 .2 and symmetry, we may assume that $r_{2}(E-$ $\left.\left\{a_{1}, b_{1}, c_{1}\right\}\right)=2$. Let $X=E-\left\{a_{1}, b_{1}, c_{1}\right\}$ and $Y=X-B_{1}$. Since each transversal in $\left\{a_{1}, b_{1}, c_{2}, c_{3}\right\}$ is $M_{1}$-independent and each transversal in $\left\{a_{2}, a_{3}, b_{2}, b_{3}, c_{1}\right\}$ is $M_{2}$-independent, there is no $M_{3}$-independent transversal in $X$; thus $r_{3}(X)=2$. Similarly, since each transversal in $\left\{a_{2}, a_{3}, b_{1}, c_{2}, c_{3}\right\}$ is $M_{2}$-independent and each transversal in $\left\{a_{2}, a_{3}, b_{2}, b_{3}, c_{1}\right\}$ is $M_{3}$-independent, we conclude that $r_{1}\left(Y \cup\left\{a_{1}\right\}\right)=2$. Without loss of generality, $a_{2} \notin \operatorname{cl}_{1}(Y)$, and so both $\left\{a_{2}, b_{2}, c_{2}\right\}$ and $\left\{a_{2}, b_{3}, c_{3}\right\}$ are $M_{1}$-independent. This means that $\left\{a_{1}, b_{1}, c_{2}\right\}$ and $\left\{a_{1}, b_{1}, c_{3}\right\}$ are $M_{2}$-dependent, for otherwise we again have three disjoint transversals that are independent in their respective matroids. Thus $r_{2}\left(\left\{a_{1}, b_{1}, c_{2}, c_{3}\right\}\right)=2$ and $E-\left\{c_{1}\right\}$ is an eight-point line in $M_{2}$, which is a contradiction.

Assume $B_{2}$ is dependent in $M_{1}$. Thus, some line $L$ in $M_{1}$ contains $B_{2}$; we may assume that $L$ also contains $a_{1}$ and $c_{1}$, since any nontrivial line contains a transversal. Also, there must be some element $a_{3}$, say, of $B_{1}$ that is not in $\operatorname{cl}_{1}(L)$. But then no transversal containing both $a_{3}$ and $c_{1}$ is dependent in $M_{1}$, leading to a contradiction by 2.1.3. Thus each of $B_{1}, B_{2}$, and $B_{3}$ is independent in all three matroids. This provides additional symmetry since we may now permute $\left(B_{1}, B_{2}, B_{3}\right)$.

Suppose next that $M_{1}$ contains a five- (or six-) point line $L$. By the conclusion of the last paragraph, we may assume that $a_{1}, b_{1}, b_{2}, c_{1}, c_{2} \in$ $L$ and that $a_{3} \notin \operatorname{cl}_{1}(L)$. Now, since there is an $M_{1}$-dependent transversal containing $a_{3}, b_{1}$, we have that $\left\{a_{3}, b_{1}, c_{3}\right\}$ must be $M_{1}$-dependent. Likewise $\left\{a_{3}, b_{2}, c_{3}\right\}$ is $M_{1}$-dependent, and thus $r_{1}\left(\left\{a_{3}, b_{1}, b_{2}, c_{3}\right\}\right)=2$, contradicting the fact that $a_{3} \notin \operatorname{cl}_{1}(L)$. Hence, none of $M_{1}, M_{2}$, and $M_{3}$ have lines containing more than four points. 
We suppose now that the transversal $\left\{a_{3}, b_{3}, c_{3}\right\}$ is $M_{2}$-independent and $M_{3}$-dependent. Since $r_{1}\left(E-\left\{a_{3}, b_{3}, c_{3}\right\}\right)=3$, we may assume that $\left\{a_{1}, b_{1}, c_{1}\right\}$ is $M_{1}$-independent, and also that $r_{3}\left(\left\{a_{2}, b_{2}, c_{2}\right\}\right)=2$ for otherwise we have the required disjoint bases. Now, at most one of $a_{3}, b_{3}$, and $c_{3}$ may be contained in $\mathrm{cl}_{3}\left(\left\{a_{2}, b_{2}, c_{2}\right\}\right)$, so without loss of generality both $\left\{a_{2}, b_{3}, c_{2}\right\}$ and $\left\{a_{3}, b_{2}, c_{2}\right\}$ are $M_{3}$-independent. Then $\left\{a_{3}, b_{2}, c_{3}\right\}$ and $\left\{a_{2}, b_{3}, c_{3}\right\}$ are both $M_{2}$-dependent. The transversal $\left\{a_{2}, b_{2}, c_{3}\right\}$ must now be $M_{2}$-independent, for otherwise we get a line in $M_{2}$ containing $\left\{a_{3}, b_{3}, c_{3}\right\}$. Thus $r_{3}\left(\left\{a_{3}, b_{3}, c_{2}\right\}\right)=2$, and further $r_{3}\left(\left\{a_{3}, b_{3}, c_{2}, c_{3}\right\}\right)=2$. Then both of $\left\{a_{2}, b_{2}, c_{3}\right\}$ and $\left\{a_{3}, b_{2}, c_{3}\right\}$ are $M_{3}$-independent, for otherwise there is a line in $M_{3}$ that contains $E-\left\{a_{1}, b_{1}, c_{1}\right\}$. But now $r_{2}\left(\left\{a_{3}, b_{3}, c_{2}\right\}\right)=r_{2}\left(\left\{a_{2}, b_{3}, c_{2}\right\}\right)=2$. This, together with the dependence of $\left\{a_{3}, b_{2}, c_{3}\right\}$ and $\left\{a_{2}, b_{3}, c_{3}\right\}$ in $M_{2}$, further implies that $\left\{a_{3}, b_{3}, c_{3}\right\}$ is $M_{2}$-dependent, which is a contradiction.

From now on, we may assume that $M_{1}, M_{2}$, and $M_{3}$ are the same matroid $M$, since they share the same set of independent transverals. Suppose that $M$ contains the four-point line $\left\{a_{3}, b_{3}, c_{2}, c_{3}\right\}$. Without loss of generality, we may assume that $\left\{a_{1}, b_{1}, c_{1}\right\}$ is independent in $M$, but then both $\left\{a_{2}, b_{3}, c_{3}\right\}$ and $\left\{a_{3}, b_{2}, c_{2}\right\}$ are also independent in $M$, so we are done.

Thus, the rank-2 flats in $M$ each contain at most three points. Let $\left\{a_{3}, b_{3}, c_{3}\right\}$ be a dependent transversal of $M$. By 2.1.1, the set $\left\{a_{3}, b_{2}, c_{1}, c_{2}\right\}$ contains a transversal that is independent in $M$. Suppose without loss of generality that $\left\{a_{3}, b_{2}, c_{2}\right\}$ is such a transversal. Then, again by 2.1.1, the set $\left\{a_{1}, a_{2}, b_{1}, c_{1}\right\}$ contains an $M$-independent transversal, $\left\{a_{1}, b_{1}, c_{1}\right\}$ say. Finally, $\left\{a_{2}, b_{3}, c_{3}\right\}$ is also independent, for otherwise we get a four-point line, and we have the three required transversals.

\section{Proof of Theorem 1.1}

Before proving Theorem 1.1, we require two further lemmas. These allow us to apply induction with Theorem 2.1 as the base case. Let $\mathcal{B}(M)$ denote the set of bases of a matroid $M$.

Lemma 3.1. Let $B_{1} \in \mathcal{B}\left(M_{1}\right), B_{2} \in \mathcal{B}\left(M_{2}\right)$ be disjoint bases of rank-n paving matroids on the same ground set, where $n \geq 3$. Let $X$ be a two-element subset of $B_{1}$. Then there is some $x \in X, y \in B_{2}$ such that $\left(B_{1}-x\right) \cup y \in \mathcal{B}\left(M_{1}\right)$ and $\left(B_{2}-y\right) \cup x \in \mathcal{B}\left(M_{2}\right)$.

Proof. Since $M_{1}, M_{2}$ are paving matroids, $\left(B_{1}-X\right) \cup y$ is $M_{1^{-}}$ independent for all $y \in B_{2}$. Suppose that both $\left(B_{1}-x\right) \cup y$ and $\left(B_{1}-x^{\prime}\right) \cup y$ are circuits in $M_{1}$, where $x, x^{\prime}$ are distinct elements of $X$. Then by circuit elimination, $B_{1}$ is also a circuit of $M_{1}$. Hence for each 
$y \in B_{2}$, at least one of $\left(B_{1}-x\right) \cup y$ and $\left(B_{1}-x^{\prime}\right) \cup y$ must be a basis of $M_{1}$.

Let $y_{1}, y_{2}, y_{3}$ be distinct elements of $B_{2}$. Then without loss of generality $\left(B_{1}-x\right) \cup y_{1},\left(B_{1}-x\right) \cup y_{2} \in \mathcal{B}\left(M_{1}\right)$. But then either $\left(B_{2}-y_{1}\right) \cup x$ or $\left(B_{2}-y_{2}\right) \cup x$ is a basis of $M_{2}$, so we are done.

Lemma 3.2. Let $B_{1}, \ldots, B_{n}$ be disjoint sets of size $n \geq 3$ and let $M_{1}, \ldots, M_{n}$ be rank-n paving matroids on $\bigcup_{i} B_{i}$ such that $B_{i}$ is a basis of $M_{i}$ for each $i \in\{1, \ldots, n\}$. Then there is an ordering of the elements of $B_{1}$ as $a_{1}, \ldots, a_{n}$ and a transversal $\left\{b_{2}, \ldots, b_{n}\right\}$ of $B_{2}, \ldots, B_{n}$ such that for all $j \in\{2, \ldots, n\}$, the set $\left(B_{1}-\left\{a_{2}, \ldots, a_{j}\right\}\right) \cup\left\{b_{2}, \ldots, b_{j}\right\}$ is a basis of $M_{1}$ and $\left(B_{j}-b_{j}\right) \cup a_{j}$ is a basis of $M_{j}$.

Proof. For $j=2$, the lemma follows immediately from Lemma 3.1. Suppose now that the lemma holds for some $j \in\{2, \ldots, n-1\}$, so that $B^{\prime}=\left(B_{1}-\left\{a_{2}, \ldots, a_{j}\right\}\right) \cup\left\{b_{2}, \ldots, b_{j}\right\} \in \mathcal{B}\left(M_{1}\right)$. Then $\left|B_{1} \cap B^{\prime}\right| \geq 2$, and so by Lemma 3.1 there is some element $a_{j+1} \in B_{1} \cap B^{\prime}$ and some $b_{j+1} \in B_{j+1}$ such that $\left(B^{\prime}-a_{j+1}\right) \cup b_{j+1} \in \mathcal{B}\left(M_{1}\right)$ and $\left(B_{j+1}-b_{j+1}\right) \cup$ $a_{j+1} \in \mathcal{B}\left(M_{j+1}\right)$, thus proving the lemma.

Lemma 3.2 is stated for $j \in\{2, \ldots, n\}$ to simplify the induction process. We only need the result for $j=n$ to prove main theorem of this paper.

Proof of Theorem 1.1. Assume that the theorem is true for some $m \geq$ 3 , and take $n=m+1$. Let $B_{1}=\left\{a_{1}, \ldots, a_{n}\right\}$ and $b_{i} \in B_{i}$ for each $i \in$ $\{2, \ldots, n\}$. By Lemma 3.2 we may assume that $A_{1}=\left\{a_{1}, b_{2}, \ldots, b_{n}\right\}$ is a basis of $M_{1}$ and that $B_{i}^{\prime}=\left(B_{i}-b_{i}\right) \cup a_{i}$ is a basis of $M_{i}$ for each $i \in\{2, \ldots, n\}$.

Now let $X=E-\left(B_{1} \cup A_{1}\right)$ and $M_{i}^{\prime}=\left(M_{i} / a_{i}\right) \mid X$ for each $i \in$ $\{2, \ldots, n\}$. Then each $M_{i}^{\prime}$ is a rank- $m$ paving matroid having $B_{i}-b_{i}$ as a basis. By our induction hypothesis, there are disjoint transversals $A_{2}^{\prime}, \ldots, A_{n}^{\prime}$ of these $m$ bases such that $A_{i}^{\prime}$ is a basis of $M_{i}^{\prime}$. But then $A_{i}=A_{i}^{\prime} \cup a_{i}$ is a basis of $M_{i}$ for each $i \in\{2, \ldots, n\}$. Moreover, the bases $A_{1}, \ldots, A_{n}$ are disjoint transversals of $B_{1}, \ldots, B_{n}$ as required.

\section{ACKNOWLEDGEMENTS}

The authors wish to thank the anonymous referees for their helpful comments.

\section{REFERENCES}

[1] Aharoni, R. \& Berger, E., The intersection of a matroid and a simplicial complex, preprint. 
[2] Chan,W., An exchange property of matroid, Discrete Math. 146 (1995), 299302.

[3] Chow, T., On the Dinitz conjecture and related conjectures, Discrete Math. 145 (1995), 73-82.

[4] Drisko, A.A., On the number of even and odd Latin squares of order $p+1$, Advances in Math. 128 (1997), 20-35.

[5] Drisko, A.A., Proof of the Alon-Tarsi conjecture for $n=2^{r} p$, Electron. J. Combin. 5 (1998), R28.

[6] Huang, R. \& Rota, G.-C., On the relations of various conjectures on Latin squares and straightening coefficients, Discrete Math. 128 (1994), 225-236.

[7] Oxley, J.G., Matroid Theory. Oxford University Press, New York (1992).

[8] Ponomarenko, V., Reduction of jump systems, Houston J. Math. 30 (2004), 27-33.

[9] Wild, M., On Rota's problem about $n$ bases in a rank $n$ matroid, Advances in Math. 108 (1994), 336-345.

DEPaRTMENT OF COMBINATORICS AND OPTIMIZATION, UNIVERSITY OF WATERloo, Waterloo, Canada

Department of Mathematics and Statistics, University of CanterBury, Christchurch, NeW Zealand

E-mail address: pjh96@student.canterbury.ac.nz 